\title{
Gastropatía aguda hemorrágica y epistaxis, marcadores clínicos en el medio rural de la variante B.1617.2 (delta) del SARS-CoV-2
}

\author{
Hemorrhagic gastropathy acute and epistaxis, rural areas clinical markers of SARS-CoV-2 \\ variant B.1617.2 (delta)
}

\section{María E. Aguilar-Aldrete', José Domínguez-Rodas², Carlos E. Cabrera-Pivaral', Nemesio Villa-Ruano, Sabina López-Toledo ${ }^{4}$, Nory O. Dávalos-Rodríguez ${ }^{5}$, Ana R. Rincón-Sánchez ${ }^{4}$, Erick Ruiz-Balderas ${ }^{6}$ y} Sergio A. Ramírez-García ${ }^{5 *}$

${ }^{1}$ Departamento de Salud Pública, Centro Universitario de Ciencias de la Salud (CUCS), Universidad de Guadalajara, Jalisco; ${ }^{2}$ Servicios Médicos Profesionales, Particulares, A.C. Miahuatlán de Porfirio Díaz, Oaxaca; ${ }^{3}$ Centro de Transferencia Tecnológica, Benemérita Universidad Autónoma de Puebla, Puebla de los Ángeles, Puebla; ${ }^{4}$ CB-Xpert Laboratorio de Patología Clínica, Miahuatlán de Porfirio Díaz, Oaxaca; ${ }^{5}$ Departamento de Biología Molecular y Genómica, Instituto de Biología Molecular y Terapia Génica, CUCS, Universidad de Guadalajara, Jalisco; ${ }^{6}$ Departamento de Biología Molecular y Genómica, Instituto de Nutrición, Universidad de la Sierra Sur, SUNEO, Miahuatlán de Porfirio Díaz, Oaxaca. México

\section{A los editores:}

Los artículos de Ramírez-García et al. ${ }^{1}$, Carrillo-Esper et al. ${ }^{2}$ y Parra-Romero et al. ${ }^{3}$ son básicos para el abordaje de la coagulopatía y la enfermedad vascular por COVID-19, sobre todo en el contexto de la variante B.1617.2 (delta) ${ }^{4}$, debido a su probable relación con trastornos hemorreológicos. En este tópico, recientemente se ha reportado Enfermedad Vascular Cerebral (EVC) y trombocitopenia en los pacientes con COVID-19. Nosotros detectamos tres casos con variante delta, con edades de 71, 86 y 96 años, con gastropatía aguda hemorrágica, PCR-genómico negativo para Helicobacter pilory, con trombocitopenia, valores séricos bajos de factor de Von Willebrand, dímero $D$, ferritina e interleucina 6 , y sin factores genéticos de trombofilia. Además, en 72 de 76 nuevos casos de jóvenes (6-25 años de edad) con la variante delta en la Sierra Sur de Oaxaca, de enero a julio de 2021, se observó epistaxis sin otra etiología. Por otro lado, en el laboratorio de patología clínica, de un total de 45 muestras analizadas de pacientes positivos para $\mathrm{CO}$ VID-19 variante delta mediante qRT-CR múltiplex, 42 correspondían a casos con epistaxis. Estos datos podrían ser útiles como marcadores clínicos para el diagnóstico clínico temprano.

Basándonos en lo expuesto, consideramos de suma importancia evaluar los marcadores moleculares de trombofilia, por la relevancia en la anticoagulación. Se postula que algunos genotipos de la variante delta producen una macroangiopatía aguda hemorrágica asociada a tromboastenia y trombocitopenia, mientras que las otras cepas del SARS-CoV-2 producen microangiopatía inflamatoria aguda trombótica. Estos hallazgos deben corroborarse en estudios masivos de réplica de vigilancia genómica por secuenciación masiva.

La trombocitopatía asociada a la variante delta puede deberse a un defecto en la agregación o adherencia de las plaquetas entre sí y al endotelio vascular, debido a que algunas variantes del virus pueden tener mayor afinidad por las glucoproteínas Ila/lllb y llb/Illa plaquetarias $^{5}$.

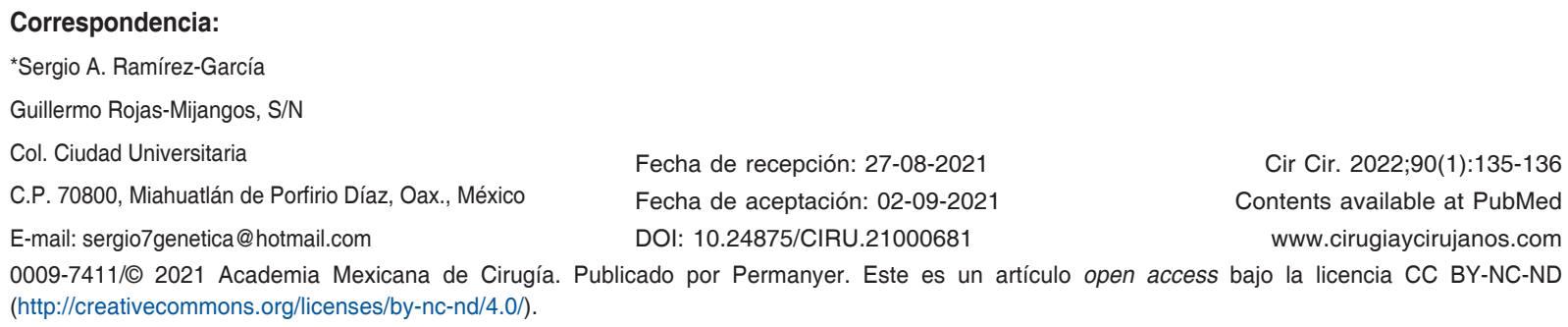

Cir Cir. 2022;90(1):135-136

Contents available at PubMed www.cirugiaycirujanos.com 0009-7411/@ 2021 Academia Mexicana de Cirugía. Publicado por Permanyer. Este es un artículo open access bajo la licencia CC BY-NC-ND (http://creativecommons.org/licenses/by-nc-nd/4.0/). 


\section{Agradecimientos}

Los autores agradecen a la Fundación Mexicana de Enfermedades Genéticas y Medicina Genómica, AC, por el financiamiento de los estudios moleculares de trombofilia.

\section{Financiamiento}

CB-Xpert Laboratorio de Patología Clínica, Miahuatlán de Porfirio Díaz, Oaxaca, México.

\section{Conflicto de intereses}

Los autores declaran no tener conflicto de intereses.

\section{Responsabilidades éticas}

Protección de personas y animales. Los autores declaran que para esta investigación no se han realizado experimentos en seres humanos ni en animales.
Confidencialidad de los datos. Los autores declaran que han seguido los protocolos de su centro de trabajo sobre la publicación de datos de pacientes.

Derecho a la privacidad y consentimiento informado. Los autores han obtenido el consentimiento informado de los pacientes y/o sujetos referidos en el artículo. Este documento obra en poder del autor de correspondencia.

\section{Bibliografía}

1. Ramírez-García SA, García-Cruz D, Dávalos NO, López S, Landeta S, Domínguez J, et al. Alteraciones de la coagulación y marcadores de trombofilia en un paciente con SARS-CoV-2, diabetes tipo 2, hipotiroidismo y flebitis de miembro pélvico izquierdo. Cir Cir 2021:89:559-62.

2. Carrillo-Esper R, Melgar-Bieberach RE, Jacinto SA, Tapia M Campa AN. Alteraciones de la coagulación en COVID-19. Cir Cir. 2020;88:787-93.

3. Parra-Romero G, Mar-Álvarez A, Navarro-Olvera JL, Hernández-Valencia AF, Aguado-Carrillo G, Carrillo-Ruiz JD. Hemorrhagic stroke associated to COVID-19 infection in México General Hospital. Cir Cir. 2021;89:435-42.

4. Aleem A Akbar Samad AB, Slenker AK. Emerging variants of SARSCoV-2 and novel therapeutics against coronavirus (COVID-19). StatPearIs [Internet]. Treasure Island (FL): StatPearls Publishing; 2021 Jan.

5. Flores LJ, Ramírez-García SA, Candelario G. Acute pyelonephritis: the clot unfolds, evidence than supports complex nature of the renal vein thrombosis; therapeutical implications. Brain Disord Ther. 2015;4:168. 